\title{
A CHARACTERIZATION OF ABELIAN GROUPS
}

\author{
L. BRAILOVSKY
}

(Communicated by Ronald M. Solomon)

\begin{abstract}
Let $G$ be a group and let $k>2$ be an integer such that $\left(k^{3}-k\right)<$ $|G| / 2$ if $G$ is finite. Suppose that the condition $\left|A^{2}\right| \leq k(k+1) / 2$ is satisfied by every $k$-element subset $A \subseteq G$. Then $G$ is abelian.
\end{abstract}

\section{INTRODUCTION}

The problem considered in this paper comes from a class of problems concerning the structure of groups that satisfy the small squaring property on $k$-sets. This notion was introduced in [BFP] and is as follows. Let $G$ be a group and $k>1$ an integer. For a finite subset $A \subseteq G$ set

$$
A^{2}=\left\{a_{i} a_{j} \mid a_{i}, a_{j} \in A\right\} .
$$

We say that $G$ has the small squaring property on $k$-sets (and we write $G \in$ $D S(k))$ if $\left|A^{2}\right|<k^{2}$ for any subset $A \subseteq G$ of order $k$. Obviously, if $G$ is abelian then $G \in D S(k)$ for any $k>1$, and the small squaring property may be regarded as a generalization of the commutativity property.

The classification of nonabelian $D S(k)$ groups was performed for $k=2$ in [Fr]. For $k=3$ the classification was basically done in [BFP], and it was completed and generalized for infinite groups in [LM].

For the general $k$ it was shown by Neumann (see [HLM]) that if $G \in D S(k)$ then $G$ has normal subgroups $M, N$ with $1 \leq M<N \leq G$ such that $|M|$ and $|G / N|$ are bounded above by a function of $k$ and $N / M$ is abelian, i.e., $G$ is "finite by abelian by finite". This result was used in [HLM] to show that a group $G \in D S(k)$ if and only if $G$ is either "abelian by finite" or "finite by elementary abelian 2-group".

However, if $G$ is abelian then not only $G \in D S(k)$ but also

$$
\left|A^{2}\right| \leq k(k+1) / 2
$$

for any subset $A \subseteq G$ of order $k$. In this paper we show that this "very small" squaring property actually characterizes abelian groups.

Received by the editors July 1, 1991.

1991 Mathematics Subject Classification. Primary 20A05, 20 E34.

The content of this paper corresponds to a part of the author's Ph.D. thesis research carried out in Tel-Aviv University under the supervision of Professors G. Freiman and M. Herzog. 


\section{THEOREM}

We will prove the following

Theorem. Let $G$ be a group and let $k>2$ be an integer such that $\left(k^{3}-k\right)<$ $|G| / 2$ if $G$ is finite. Suppose that the condition $\left|A^{2}\right| \leq k(k+1) / 2$ is satisfied by every $k$-element subset $A \subseteq G$. Then $G$ is abelian.

The basic idea of the proof is contained in the following

Lemma. If $G$ satisfies the conditions of the theorem then

$$
\left|B^{2}\right| \leq l(l+1) / 2
$$

for any l-element subset $B \subseteq G$, where $2 \leq l<k$.

Proof. The proof is by induction on $l$. Suppose that $\exists B \subseteq G,|B|=k-1$ such that $\left|B^{2}\right|>(k-1) k / 2$. Obviously, we may assume that $\left|B^{2}\right| \leq k(k+1) / 2$. Let $B=\left\{b_{1}, \ldots, b_{k-1}\right\}$.

Denote $K_{1}^{B}=\left\{x \in G \mid x B \cap B^{2} \neq \varnothing\right\}$ and $K_{2}^{B}=\left\{x \in G \mid B x \cap B^{2} \neq \varnothing\right\}$. Since $x \in K_{1}^{B}$ iff $\exists b_{i}, b_{j}, b_{k} \in B$ such that $x=\left(b_{j} b_{k}\right) b_{i}^{-1}$, we obtain that

$$
\left|K_{1}^{B}\right| \leq\left|B^{2}\right| \cdot|B| \leq(k(k+1) / 2)(k-1)=\left(k^{3}-k\right) / 2 .
$$

Similarly, $\left|K_{2}^{B}\right| \leq\left(k^{3}-k\right) / 2$, so

$$
\left|K_{1}^{B} \cup K_{2}^{B}\right| \leq k^{3}-k \text {. }
$$

Choose $x \in G$ such that $x \notin K_{1}^{B} \cup K_{2}^{B}$ and let $A=B \cup\{x\},|A|=k$. Consider $A^{2}=B^{2} \cup x B \cup B x \cup\left\{x^{2}\right\}$. By the choice of $x$ we have $\left|B^{2} \cup x B\right|=$ $\left|B^{2}\right|+(k-1)$. Suppose that $x B \neq B x$. Then

$$
\left|A^{2}\right| \geq\left|B^{2}\right|+k>((k-1) k / 2)+k=k(k+1) / 2,
$$

which is a contradiction.

Thus, we have shown that

$$
x B=B x \quad \forall x \in G \backslash\left\{K_{1}^{B} \cup K_{2}^{B}\right\} .
$$

Denote $N_{G}(B)=\left\{x \in G \mid x B x^{-1}=B\right\}$. Obviously, $N_{G}(B) \leq G$ and, in view of (2) and (3), $\left|G \backslash N_{G}(B)\right| \leq k^{3}-k$. If $G$ is infinite then $N_{G}(B)=G$, since $\left|G \backslash N_{G}(B)\right|<\infty$. If $G$ is finite, then again $N_{G}(B)=G$, since $|G| / 2>\left(k^{3}-k\right)$ by the condition of the theorem. Thus, $g B g^{-1}=B \forall g \in G$. In particular, $b_{i} B=B b_{i}$ for all $b_{i} \in B$, so the sets of elements of the $i$ th row and the $i$ th column of the multiplication table of $B \times B$ coincide for $i=1, \ldots, k-1$. Hence $\left|B^{2}\right| \leq(k-1) k / 2$, which is a contradiction.

We proceed now to the proof of the theorem. Suppose that a group $G$ satisfies the condition of the theorem and $G$ is nonabelian. By the lemma, condition (1) holds for any $l$-element subset $B \subseteq G$, where $2 \leq l<k$. In particular, it holds for $l=2$, which means that $G \in D S(2)$. Then, as it is shown in [Fr], $G=Q_{8} \times E$, where $Q_{8}$ is the quaternion group of order 8 and $E$ is an elementary abelian 2-group. Take $i, j, k \in Q_{8}$ to be the standard generators of the quaternion group and consider the 3-element set

$$
B=\{(i, 1),(j, 1),(k, 1)\} \subseteq G .
$$


Then $\left|B^{2}\right|>6$, which is a contradiction, since, by the lemma, condition (1) must hold for any 3-element subset of $G$ as well. Thus $G$ has to be abelian and the theorem is proved.

\section{REFERENCES}

[BFP] Ja. G. Berkovich, G. A. Freiman, and Cheryl E. Praeger, Small squaring and cubing properties for finite groups, Bull. Austral. Math. Soc. 44 (1991), 429-450.

[Fr] G. A. Freiman, On two- and three-element subsets of groups, Aequationes Math. 22 (1981), 140-152.

[HLM] M. Herzog, P. Longobardi, and M. Maj, On a combinatorial problem in group theory (to appear).

[LM] P. Longobardi and M. Maj, The classification of groups with the small squaring property on 3-sets (to appear).

School of Mathematical Sciences, Raymond and Beverly Sackler faculty of Exact SCIENCES, Tel-Aviv University, RAMAT-Aviv, 69978 ISRAel

E-mail address: brail@math.tau.ac.il 